Recebido em: 27 Maio $2017 \quad$ Aprovado em: 10 Jul. 2017 Publicado em: 12 Set. 2017 DOI: https://doi.org/10.18554/rt.v10i1.2162

v. 10 n. 1 - Jan. / Jun. 2017

\title{
ORIENTACIÓN DE META Y CLIMA MOTIVACIONAL PERCIBIDO EN LAS CLASES DE EDUCACIÓN FÍSICA
}

\author{
ORIENTAÇÃO DE META E CLIMA MOTIVACIONAL PERCEBIDO EM AULAS DE \\ EDUCAÇÃO FÍSICA
}

\section{GOAL ORIENTATION AND PERCEIVED MOTIVATIONAL CLIMATE IN PHYSICAL EDUCATION CLASSES}

\author{
Diana Carolina Quintana Moreno \\ Universidad Pedagógica y Tecnológica de Colombia - UPTC / Colômbia \\ E-mail: diana.agdd@gmail.com \\ Iliana Paola Quintana Moreno \\ Universidad El Bosque - UELBOSQUE / Colômbia \\ E-mail: iquintana@unbosque.edu.co \\ Thiago Henrique Barnabé Corrêa \\ Universidade Federal do Triângulo Mineiro - UFTM / Brasil \\ E-mail: correa.uftm@gmail.com
}

\begin{abstract}
RESUMEN
Con el fin de analizar la relación entre las orientaciones de meta y el clima motivacional percibido por los estudiantes en las clases de Educación Física en colegios públicos y privados de Chiquinquirá, Boyacá (Colombia). En este estudio de tipo descriptivo-correlacional de corte trasversal; se tomó una muestra de tipo no probabilística la cual estuvo conformada por 378 sujetos (178 hombres y 200 mujeres) de grado noveno, a quienes se les aplico el Cuestionario de Orientación al Ego y a la Tarea, (TEOSQ) y el Cuestionario de Orientación al Aprendizaje y al Rendimiento en las Clases de Educación Física, (LAPOPECQ). El procesamiento de los datos se realizó mediante el software estadístico SPSS 13 en su versión estudiantil. Los resultados mostraron una relación significativa entre la orientación a la tarea y al clima motivacional orientado al aprendizaje, lo que quiere decir que los estudiantes evalúan su proceso de formación basándose en ellos mismos y perciben que el clima motivacional orientado por el profesor está dirigido más hacia la tarea que hacia el ego.
\end{abstract}

PALABRAS-CLAVE: Psicología deportiva. Motivación y Aprendizaje. Educación Física.

\section{RESUMO}

Este artigo tem como fim analisar a relação entre orientações de meta e clima motivacional percebido pelos alunos em aulas de educação física em escolas públicas e privadas em Chiquinquirá, Boyacá (Colômbia). Neste estudo de tipo descritivo-correlacional de corte transversal, utilizou-se uma amostra não-probabilística, que consistiu de 378 individuos (178 homens e 200 mulheres) da nona série, onde aplicou-se um questionário de orientação (TEOSQ) e um questionário de orientação de aprendizagem e desempenho nas aulas de Educação física (LAPOPECQ). O processamento dos dados foi realizado usando o software estatístico SPSS 13 em sua versão para estudantes. Os resultados mostraram uma relação significativa entre a orientação para a tarefa e a clima motivacional, o que significa que os alunos podem avaliar seu processo de formação com base em si e perceber que o clima motivacional orientado pelo professor está mais dirigido para a tarefa que para o ego.

PALAVRAS-CHAVE: Psicologia do Esporte. Motivação e Aprendizagem. Educação Física. 


\begin{abstract}
In order to analyze the relationship between the goal orientations and the motivational climate perceived by the students in the Physical Education classes in public and private schools in Chiquinquirá, Boyacá, Colombia. In this cross-sectional descriptive-correlational study; A sample of non-probabilistic type was taken which was formed by 378 subjects (178 men and 200 women) of ninth degree, who were given the Ego and Homework Guidance Questionnaire (TEOSQ) and the Guidance Questionnaire Learning and Performance in the Physical Education Classes, (LAPOPECQ). Data processing was performed using the statistical software SPSS 13 in its student version. The results showed a significant relationship between task orientation and learning-oriented motivational climate, which means that students evaluate their learning process on the basis of themselves and perceive that the teacher-oriented motivational climate is more directed towards The task that led to the ego.
\end{abstract}

KEYWORDS: Sports psychology. Motivation and Learning. Physical education.

\title{
INTRODUCCIÓN Y APORTES TEÓRICOS ADOPTADOS
}

A través de la historia la actividad física (A.F) ha sido formativa, educativa, competitiva, recreativa, preventiva, atrayente y popular en nuestro medio; no solo porque es una actividad recreativa, sino porque contribuye con la mejora de la salud y el bienestar del individuo. En este sentido, González (2007) menciona que algunos de los beneficios físicos y psicológicos de la actividad deportiva son en primera instancia, el aumento y mejora de la fuerza, la velocidad, la resistencia, la flexibilidad, la coordinación, el equilibrio y la estructura física del estudiante, mientras que el segundo, puede atenuar las respuestas fisiológicas al estrés, mejora la autonomía y la autoestima del estudiante, y ejerce una mejor motivación hacia el aprendizaje de la actividad deportiva.

En Colombia la Ley 181 del 1995, expedida por el Congreso de La República, dicta las disposiciones para el fomento del deporte, la recreación, el aprovechamiento del tiempo libre y la Educación Física, al igual orienta las responsabilidades para la investigación en el área, buscando promover la producción científica y el desarrollo de programas nacionales de mejoramiento de la condición física.

Al respecto Madrigal y Urrego (2013), en su estudio muestran una recopilación de las investigaciones realizadas sobre educación física, recreación y deporte, encontrando la necesidad imperiosa de continuar investigando al interior del área, para revisar los contenidos tradicionales y los tratamientos instrumentales de las actividades físicas. Lo anterior también en concordancia con la tendencia internacional que impone un compromiso para el desarrollo investigativo en educación.

A propósito Rodríguez et al. (2011) aseguran que al ejercicio físico y al deporte se les ha asignado una función social, asociada a la preservación y desarrollo de la salud en el ser humano. Por lo que en la actualidad la instauración de estilos de vida no activos, deben 
convertirse en un objetivo esencial de la política educativa, debido a las conductas pasivas, no activas y sedentarias de la población escolar.

Más cuando los efectos positivos de la práctica físico-deportiva en la población escolar, no corresponden con la frecuencia esperada de estas prácticas. Según Rodríguez et al. (2011) numerosas investigaciones señalan un descenso progresivo de la práctica físicodeportiva a medida que se va pasando de la infancia a la adolescencia.

Lo anterior puede explicar la necesidad de contar con estudios que identifiquen las relaciones entre las orientaciones de meta y el clima motivacional percibido en estudiantes colombianos, con el fin de establecer estrategias que ayuden al maestro en el proceso enseñanza-aprendizaje y a fomentar en el estudiante estilos de vida activos que se continúen en la edad adulta, principalmente como un factor que contribuya a la salud y su bienestar, a través del compromiso y de un rol activo en las clases de Educación Física.

Las investigaciones que han girado en torno a estas variables dan a conocer la teoría de orientación a la meta, como un fundamento teórico útil que permite la comprensión del comportamiento de logro, el aprendizaje, el rendimiento académico y deportivo de los estudiantes, desde un enfoque social cognitivo, a la vez que realizan una presentación de la teoría en el ámbito de la educación y la actividad física (KOHLER \& REYES, 2010).

Las orientaciones de meta y/o disposicionales se caracteriza por un patrón integrado de creencias, atribuciones y afectos que se dirigen a una conducta, permitiendo explorar el interés y la implicación hacia una determinada acción, así como el propósito de desarrollar conductas que encaminen al logro de la meta (KOHLER \& REYES, 2010).

En los entornos de logro existen dos diferentes concepciones de lo que significa ser hábil, estas concepciones traen una serie de consecuencias en el orden afectivo, cognitivo y conductual. Así, los individuos tienden a asumir una de estas concepciones de habilidad o una combinación de las dos y actúan de acuerdo a ellas, las cuales han sido denominadas como la orientación a la tarea y la orientación al ego (NICHOLLS, 1984).

Una orientación a la tarea es una concepción de habilidad consistente en la búsqueda de la mejora en la ejecución de las actividades que desempeña, esforzándose por mejorar y convencido de que el éxito está en función de su mejora personal. Por otra parte, una orientación al ego es una concepción de habilidad que consiste en juzgar la competencia personal de forma comparativa, si se demuestra mayor habilidad que los demás miembros del grupo se muestran altos niveles de satisfacción (PEIRÓ, 1999; CASTILLO et., al, 2000; LLANOS, TABERNERO, 2003). 
Por otra parte, la motivación es realmente una cuestión principal dentro de la acción pedagógica. Todo profesor la quiere para sí, para sus estudiantes y sufre cuando falta. Responde a una demanda de comunicación continua y es objeto de investigaciones pedagógicas. Tras algunas clases logradas o decepcionantes, surge una reflexión sobre lo que habría podido movilizar a los estudiantes, lo que habría podido frenar u obstaculizar su compromiso (DUDA, 2001).

Utilizando el termino motivación de forma global, donde dependiendo de otros factores es percibida una clase como motivada o no motivada, mientras que por otro lado, nadie discutirá que la motivación es una realidad que afecta a personas en particular y que además, estas motivaciones pueden ser muy distintas en el mismo grupo. También la motivación puede ser percibida como algo dado, una cualidad que poseen ciertos estudiantes y otros no. La motivación, verdadero fundamento de todo comportamiento humano, es algo que se construye, que evoluciona bajo la influencia del entorno pedagógico y del propio estudiante (DEVÍS, PEIRÓ, 1992; MAYOR, CANTON, 1995; GUZMAN, GARCÍAFERRIOL, 2002).

Partiendo de la concepción de la motivación desde el ámbito educativo, definida por Ames (1992 apud ALMAGRO, SAÉNZ-LÓPEZ, GONZÁLEZ-CUTRE, MORENOMURCIA, 2011) como "un conjunto de señales implícitas, y/o explícitas, percibidas en el entorno, a través de las cuales se definen las claves de éxito y fracaso" (p. 25). El clima motivacional se desarrolla conforme a los estilos de enseñanza, la forma en que los niños se agrupan para aprender y las creencias del profesor sobre el éxito o fracaso de sus alumnos (CECCHINI, GÓNZÁLEZ, CARMONA, CONTRERAS, 2004). De esta forma es indispensable que en ámbitos como la educación física haya un clima adecuado que facilite los procesos de enseñanza-aprendizaje eficaces que impliquen tanto a la tarea como al individuo.

Resaltando dos tipos de clima motivacional; el dirigido a la tarea y el dirigido al ego. En el primero se considera primordial promover valores como la diversión, la satisfacción, el interés y la motivación intrínseca, donde se fomente el aprendizaje cooperativo y una implicación activa por parte de los individuos en el proceso; mientras que en el segundo implica una relación positiva con la orientación al ego, la afectividad negativa y sentimientos de presión, donde las recompensas son obtenidas con el rendimiento superior en comparación a otros (SÁNCHEZ-LIVIA et al., 2010).

Es por esto que el presente estudio busca analizar la relación entre las orientaciones de meta y el clima motivacional percibido de los estudiantes en las clases de educación física en 
colegios públicos y privados de Chiquinquirá, Boyacá; los resultados de este estudio se constituirá en un diagnóstico para los docentes de estas instituciones y la construcción de nuevas estrategias para la adherencia de los estudiantes hacia las clases de educación física, fomentando estilos de vida saludables, que contribuyan con su salud y bienestar a nivel físico y psicológico.

\section{METODOLOGÍA}

Este estudio es de tipo descriptivo-correlacional dentro del paradigma cuantitativo y de corte trasversal; de acuerdo con Hernández Sampieri, Fernández-Collado y Baptista (2014) se entiende como la medición del grado de relación existente entre estas dos variables, buscando analizar la relación entre las orientaciones de meta y el clima motivacional percibido de los estudiantes en las clases de Educación Física en colegios públicos y privados de Chiquinquirá, Boyacá (Colombia). De igual forma, al revestirse la investigación con el enfoque cuantitativo - a través de la aplicación de los cuestionarios validados a población colombiana por Flórez, Salguero y Márquez (2008) -, permite caracterizar los grupos identificados según el género y las variables arriba señaladas teniendo en cuenta las valoraciones de las relaciones y el grado de asociación entre las mismas.

Utilizando un muestreo no probabilístico por conveniencia el cual, según McMillan y Schumacher (2005), se caracteriza por la selección de un grupo de sujetos sobre la base de ser accesibles o adecuados de acuerdo con el criterio del investigador. De igual forma, este muestreo se determinó teniendo en cuenta los objetivos del estudio, el esquema de la investigación y su alcance. Para Hernández Sampieri, Fernández-Collado y Baptista (2014) este se realiza en investigaciones sin ánimo de generalizar los resultados a una población de referencia.

En total se contó con 378 estudiantes que cursan grado Noveno de cada institución educativa, en cuatro (4) colegios, dos de carácter público y dos de carácter privado del municipio de Chiquinquirá - Departamento de Boyacá. El 47,7\% son de género masculino y $52,9 \%$ de género femenino. En cuanto a la edad, más de la mitad de los estudiantes está entre los 14 y los 15 años de edad (82,3\%), mientras que las edades 12,18 y 19 años son las menos frecuentes con un porcentaje de $0,5 \%$ y $0,3 \%$ para las dos últimas. Respecto al estrato socioeconómico, el $44,2 \%$ de la muestra pertenece al estrato dos y el 1,3\% se ubica en el cuatro (ver Tabla 1). 
Tabla 1

Características sociodemográficas de los estudiantes participantes

\begin{tabular}{cccc}
\hline Dimensión & Carácter & Porcentaje & Frecuencia \\
\hline \multirow{2}{*}{ Sexo } & Masculino & 47,1 & 178 \\
& Femenino & 52,9 & 200 \\
& 12 años & 0,5 & 2 \\
& 13 años & 8,5 & 32 \\
\multirow{2}{*}{ Edad } & 14 años & 57,4 & 217 \\
& 15 años & 24,9 & 94 \\
& 16 años & 6,9 & 26 \\
& 17 años & 1,3 & 5 \\
& 18 años & 0,3 & 1 \\
& 19 años & 0,3 & 1 \\
\multirow{3}{*}{ Estrato } & Uno & 28,3 & 107 \\
socioeconómico & Dos & 44,2 & 167 \\
& Tres & 26,2 & 99 \\
& Cuatro & 1,3 & 5 \\
\hline
\end{tabular}

Dentro de los criterios de clasificación de los planteles, de acuerdo con los puntajes nivel superior y/o alto de las pruebas Saber-Pro del 2013. Para los estudiantes, debían ser niños y niñas matriculados en las instituciones educativas en el grado Noveno de Básica Secundaria. Y respecto a los criterios de exclusión no se tuvieron en cuenta los colegios que no aprobaron su participación en el estudio mediante la firma del consentimiento informado. Así como los estudiantes que se encontraban en incapacidad médica o suspensión académica durante el proceso de aplicación de los cuestionarios, los estudiantes que no respondieron de forma completa los cuestionarios dejando preguntas en blanco y aquellos que no contaban con la firma del consentimiento informado por parte de sus padres.

Para la recolección de la información se utilizaron dos cuestionarios uno para la valorar las orientaciones de meta y en otro para medir el clima motivacional en las clases de Educación Física. Los cuales son el TEOSQ (Task and Ego Sport Questionnaire); adaptado a la Educación Física por Walling y Duda (1995), validado al español por Peiró y cols. (2004). El cual consta de 16 ítems, donde se mide la orientación a la tarea (8 ítems) y la orientación al ego (8 ítems). Se midió su Validez a través de la medida estadística Alpha de Cronbach. Y el Cuestionario de Orientación al Aprendizaje y al Rendimiento en las Clases de Educación Física-LAPOPECQ, traducido al castellano y validado por Cervelló y Jiménez (2002). Se halla compuesto por 27 ítems y posee dos dimensiones de primer orden: percepción del clima motivacional que implica al aprendizaje (Factor A) y percepción del clima motivacional que 
implica al rendimiento (Factor R). Este es un instrumento propio del área de Educación Física y el deporte, por tanto, es de aplicación general y no tiene una limitación en cuanto al rango de edad. Para los dos cuestionarios los sujetos respondieron en una escala tipo Likert de cinco puntos, que oscila entre el 1 (nada de acuerdo) y el 5 (muy de acuerdo).

El procedimiento que se utilizó para la ejecución del presente estudio se desarrollarlo en siete (7) fases denominadas así: fase 1. Diseño de la propuesta de investigación, fase 2. Contacto con las instituciones, fase 3. Firma de consentimiento informado, fase 4. Administración de los cuestionarios, fase 5. Procesamiento de datos, fase 6. Análisis de resultado, fase 7. Propuesta de intervención.

\section{ANÁLISIS DE LOS DATOS Y RESULTADOS}

En primer lugar, el presente estudio garantizó la confiabilidad y la adecuada consistencia interna de los cuestionarios utilizados, demostrando que éstos son aptos para medir las variables de estudio en las clases de Educación Física en población escolar colombiana (ver Tabla 2). De acuerdo con Nunnaly (1978) los valores Alpha de Cronbach superiores o iguales a 0.70 indican niveles de consistencia interna apropiados para los test examinados.

Tabla 2

Alpha de Cronbach para los cuestionarios TEOSQ y LAPOPECQ

\begin{tabular}{ccc|}
\hline Instrumento & Alpha de Cronbach & No. De Ítems \\
\hline TEOSQ & 0,771 & 16 \\
LAPOPECQ. & 0,781 & 27 \\
\hline
\end{tabular}

Analizando los resultados obtenidos se ha encontrado, respecto a las orientaciones de meta, hay una relación débil y directa, lo que muestra similitud con los hallazgos de otros estudios como los realizados por Duda y Whitehead (1998), Harwood y Hardy (2001), Nicholls (1989), Roberts (2001) o Roberts, Treasure y Kavussanu (1996), quienes consideran al ego y la tarea como dimensiones independientes y ortogonales, afirmando que un sujeto puede sentirse altamente orientado a un factor $\mathrm{y}$, paralelamente, sentirse orientado a otro factor. Se deduce que en los estudiantes prevalecen los aspectos asociados con la tarea antes que aquellos asociados con el ego (ver Tabla 3 ). 
Tabla 3

Estadísticas descriptivas para el ego y la tarea

\begin{tabular}{lrr}
\hline \multicolumn{1}{c}{ Estadísticos Descriptivos } & $\begin{array}{c}\text { Factor1 } \\
\text { ego }\end{array}$ & $\begin{array}{r}\text { Factor2 } \\
\text { Tarea }\end{array}$ \\
\hline Media & 3,3502 & 4,2040 \\
Desviación Estándar &, 81935 &, 56601 \\
Mínimo & 1,00 & 1,88 \\
Máximo & 5,00 & 5,00 \\
\hline
\end{tabular}

La Tabla 4 presenta las puntuaciones promedio, desviación estándar, el mínimo y el máximo para las dos dimensiones. La puntuación promedio más alta se obtuvo en la dimensión 1 con una media igual a 4.01, lo que está asociado a que los participantes están más significativamente inclinados al aprendizaje y un aumento significativo en este refleja un aumento moderado en el rendimiento.

Tabla 4

Estadísticos descriptivos para la dimensión 1 y dimensión 2

\begin{tabular}{lcc}
\hline Estadísticos Descriptivos & $\begin{array}{c}\text { Dimensión 1(A) } \\
\text { Aprendizaje }\end{array}$ & $\begin{array}{c}\text { Dimensión 2 (R) } \\
\text { Resultado }\end{array}$ \\
\hline Media & 4,0132 & 3,0431 \\
Desviación Estándar & 0,54101 & 0,64504 \\
Mínimo & 1,81 & 1,24 \\
Máximo & 4,86 & 5,00 \\
\hline
\end{tabular}

En los resultados obtenidos se evidencia que la relación entre los cuestionarios TEOSQ y LAPOPECQ. El ego guarda una relación significativa pero moderada con: la orientación al resultado, las preocupaciones de los alumnos por los errores y la orientación al resultado sin esfuerzo. Es decir, existe una relación moderada entre el ego y aspectos asociados con el resultado. Así mismo, el ego tiene una relación débil con el clima motivacional asociado al aprendizaje por el profesor y no presentar una relación significativa. Y la tarea guarda una relación significativa pero moderada con aspectos del clima motivacional asociados al aprendizaje propiciado por el profesor y en la orientación al aprendizaje de los alumnos (ver Tabla 5). 
Tabla 5

Matriz de correlaciones entre los dos cuestionarios

\begin{tabular}{|c|c|c|c|c|c|c|c|c|}
\hline & Factor & $\begin{array}{c}\text { Factor } \\
1\end{array}$ & $\begin{array}{c}\text { Factor } \\
2\end{array}$ & $\begin{array}{r}\text { Factor } \\
1 \\
\text { (A) }\end{array}$ & $\begin{array}{r}\text { Factor } \\
2 \\
\text { (A) }\end{array}$ & $\begin{array}{r}\text { Factor } \\
\text { (R) }\end{array}$ & $\begin{array}{r}\text { Factor } \\
4 \\
\text { (R) }\end{array}$ & $\begin{array}{c}\text { Factor } \\
5(\mathrm{R})\end{array}$ \\
\hline \multirow{3}{*}{$\begin{array}{c}\text { Factor } \\
1\end{array}$} & $\begin{array}{l}\text { Correlación } \\
\text { Pearson }\end{array}$ & 1 & 0,158 & 0,1 & 0,0 & 0,2 &, 3 & 0,2 \\
\hline & Sig. (2-tailled) & & 0,002 & 0,007 & 0,535 & 0,000 & 0,000 & 0,000 \\
\hline & $\mathrm{N}$ & 378 & 378 & 378 & 378 & 378 & 378 & 378 \\
\hline \multirow{3}{*}{$\begin{array}{c}\text { Factor } \\
2\end{array}$} & $\begin{array}{l}\text { Correlación } \\
\text { Pearson }\end{array}$ & $0,158 * *$ & 1 & $0,253 * *$ & $0,284 * *$ & 0,056 & $0,156^{* *}$ & $-0,061$ \\
\hline & Sig. (2-tailled) & 0,002 & & 0,000 & 0,000 & 0,277 & 0,002 & 0,240 \\
\hline & $\mathrm{N}$ & 378 & 378 & 378 & 378 & 378 & 378 & 378 \\
\hline \multirow{3}{*}{$\begin{array}{c}\text { Factor } \\
1(\mathrm{~A})\end{array}$} & ción & $0,139 * *$ & $0,253 * *$ & 1 & $0,555^{* *}$ & $0,328 * *$ & $0,339 * *$ & 0,078 \\
\hline & Sig. (2-tailled) & 0,007 & 0,000 & & 0,000 & 0,000 & 0,000 & 0,132 \\
\hline & $\mathrm{N}$ & 378 & 378 & 378 & 378 & 378 & 378 & 738 \\
\hline \multirow{3}{*}{$\begin{array}{c}\text { Factor } \\
2(\mathrm{~A})\end{array}$} & ición & 0,032 & $0,284 * *$ & $0,555 * *$ & 1 & $0,203 * *$ & $0,119^{*}$ & $-0,124 *$ \\
\hline & Sig. (2-tailled) & 0,535 & 0,000 & 0,000 & & 0,000 & 0,021 & 0,016 \\
\hline & $\mathrm{N}$ & 378 & 378 & 378 & 378 & 378 & 278 & 378 \\
\hline \multirow{3}{*}{$\begin{array}{c}\text { Factor } \\
3(\mathrm{R})\end{array}$} & $\begin{array}{l}\text { Corre } \\
\text { Pearse }\end{array}$ & $0,263 * *$ & 0,056 & $0,328 * *$ & $0,203 * *$ & 1 &, $443 * *$ & $0,383 * *$ \\
\hline & Sig. (2-tailled) & 0,000 & 0,277 & 0,000 & 0,000 & & 0,000 & 0,000 \\
\hline & $\mathrm{N}$ & 378 & 378 & 378 & 378 & 378 & 378 & 378 \\
\hline \multirow{3}{*}{$\begin{array}{c}\text { Factor } \\
4(\mathrm{R})\end{array}$} & Correlación & $0,397 * *$ & $0,156^{* *}$ & $0,339 * *$ & $0,119 *$ & $0,443 * *$ & 1 & $0,331 * *$ \\
\hline & $\begin{array}{l}\text { Pearson } \\
\text { Sig. (2-tailled) }\end{array}$ & 0,000 & 0,002 & 0,000 & 0,021 & 0,000 & & 0,000 \\
\hline & $\mathrm{N}$ & 378 & 378 & 378 & 278 & 378 & 378 & 378 \\
\hline \multirow{3}{*}{$\begin{array}{c}\text { Factor } \\
5(\mathrm{R})\end{array}$} & Correlación & $0,292 * *$ & $-0,061$ & 0,078 & $-0,124^{*}$ & $0,383 * *$ & $0,331 * *$ & 1 \\
\hline & $\begin{array}{l}\mathrm{Pe} \\
\mathrm{Si}\end{array}$ & 000 & 240 & 132 & 016 & 0.000 & 0.000 & \\
\hline & $\mathrm{N}$ & 378 & 378 & 738 & 378 & 378 & 378 & 378 \\
\hline
\end{tabular}

Respecto a los resultados obtenidos por la variable género se identificó que existen diferencias significativas entre hombres y mujeres en el factor orientación al ego, siendo el género masculino el más orientado al ego. Esto coincide con los hallazgos obtenidos en otras investigaciones realizadas por Peiró y Sanchis (2004) y por Ruíz, Piéron y Zamarripa (2011). De igual forma, se halló que los hombres se preocupan más por los errores que las mujeres; ya que estas últimas se orientan más al clima de aprendizaje por el profesor y por el estudiante, el cual puede estar relacionado con la percepción de un trato de igualdad por parte del docente, como se señala en los estudios de (Cervelló y cols., 2004; Papaioannou, 1999) como se citó en Flórez, Salguero y Márquez (2008) (ver Tabla 6). 
Tabla 6.

Diferencia de género entre los factores del TEOSQ y LAPOPECQ

\begin{tabular}{|c|c|c|c|c|c|c|}
\hline Factor & Género & $\mathbf{N}$ & Media & DT & $\mathbf{F}$ & $\mathbf{P}$ \\
\hline \multirow{2}{*}{ Orientado al Ego } & Masculino & 178 & 3,4558 & 0,79 & \multirow{2}{*}{0,27} & \multirow{2}{*}{0,01} \\
\hline & Femenino & 200 & 3,2562 & 0,83 & & \\
\hline \multirow{2}{*}{$\begin{array}{l}\text { Orientación a la } \\
\text { Tarea }\end{array}$} & Masculino & 178 & 4,1664 & 0,57 & \multirow{2}{*}{0,64} & \multirow{2}{*}{0,22} \\
\hline & Femenino & 200 & 4,2375 & 0,55 & & \\
\hline \multirow{2}{*}{$\begin{array}{l}\text { Clima Aprendizaje } \\
\text { Profesor }\end{array}$} & Masculino & 178 & 3,7801 & 0,63 & \multirow{2}{*}{2,01} & \multirow{2}{*}{0,04} \\
\hline & Femenino & 200 & 3,9007 & 0,54 & & \\
\hline \multirow{2}{*}{$\begin{array}{l}\text { Clima Aprendizaje } \\
\text { Estudiantes }\end{array}$} & Masculino & 178 & 4,1077 & 0,63 & \multirow{2}{*}{0,00} & \multirow{2}{*}{0,03} \\
\hline & Femenino & 200 & 4,2492 & 0,61 & & \\
\hline \multirow{2}{*}{$\begin{array}{l}\text { Orientaciones } \\
\text { Resultado } \\
\text { Estudiantes }\end{array}$} & Masculino & 178 & 3,1652 & 0,75 & \multirow{2}{*}{0,10} & \multirow{2}{*}{0,75} \\
\hline & Femenino & 200 & 3,1900 & 0,76 & & \\
\hline \multirow{2}{*}{$\begin{array}{l}\text { Preocupaciones } \\
\text { Estudiantes Errores }\end{array}$} & Masculino & 178 & 3,4483 & 0,69 & \multirow{2}{*}{2,79} & \multirow{2}{*}{0,00} \\
\hline & Femenino & 200 & 3,1600 & 0,98 & & \\
\hline \multirow{2}{*}{$\begin{array}{l}\text { Orientaciones } \\
\text { Resultado Sin } \\
\text { Esfuerzo }\end{array}$} & Masculino & 178 & 2,7528 & 0,93 & \multirow{2}{*}{5,07} & \multirow{2}{*}{0,07} \\
\hline & Femenino & 200 & 2,5683 & 1,06 & & \\
\hline
\end{tabular}

\section{CONSIDERACIONES FINALES}

Las investigaciones realizadas en Europa representan un marco de referencia que debe ser tenido en cuenta en nuevos estudios, ya que han mostrado avances significativos a nivel teórico y empírico para explicar el comportamiento de escolares relacionado con factores de motivación a nivel social, contextual y personal para reconocer los motivos subyacentes a la práctica del deporte.

De lo anterior es posible explicar que toda práctica pedagógica, sin distinción de la disciplina, debe estar soportada en la teoría. Así, los resultados diagnósticos que se realicen en las instituciones educativas encuentran una razón de ser para docentes y estudiantes. Es por esto que este trabajo representa un primer avance en el análisis de las relaciones entre las orientaciones de meta y el clima motivacional percibido en las clases de Educación Física, lo cual permitirá a los docentes reconocer factores que pueden incentivar la participación activa del estudiante en su formación, teniendo en cuenta los beneficios que estas clases proporcionan a los escolares en su preparación para su vida. 
Es por esto que el papel del docente de Educación Física se reviste de importancia en el ámbito escolar, ya que está llamado a la comprensión del comportamiento del logro, del aprendizaje, el rendimiento académico y deportivo de los estudiantes. Es importante mencionar que los resultados del estudio señalan una relación significativa entre la orientación a la tarea y al clima motivacional orientado al aprendizaje, lo cual significa que los estudiantes evalúan su proceso de formación basándose en ellos mismos y perciben que el clima motivacional orientado por el profesor está dirigido más hacia la tarea que hacia el ego, lo que puede relacionarse positivamente con los motivos para implicarse en las clases de Educación Física.

Así mismo, los resultados mostraron una relación débil entre la orientación a la tarea y los aspectos asociados con la orientación al resultado tales como: las preocupaciones de los estudiantes por los errores, la orientación al resultado de los estudiantes y la orientación al resultado sin esfuerzo; probablemente porque los estudiantes evalúan sus habilidades en las clases de Educación Física basados en el dominio de la tarea que estén ejecutando pues comprenden que, cuando logran ejecutar la tarea, están aprendiendo.

Por otra parte, entendiendo la orientación al ego como la comparación que hacen los estudiantes de su habilidad con respecto a la de sus pares dentro de las clases de Educación Física, se encuentra que el ego se relaciona de manera significativa con la orientación al resultado de los estudiantes, las preocupaciones de los estudiantes por los errores y la orientación al resultado sin esfuerzo; esta actitud puede estar más asociada a motivos de aprobación social y a la demostración de ser los mejores cuando evalúan su ejecución basados en la de sus compañeros.

Es así que los resultados establecen una relación moderada entre la orientación al ego y la orientación al resultado, lo que puede explicar que un estudiante quiera demostrar su mayor capacidad e intente recibir reconocimiento por la misma, frente a sus demás compañeros.

Respecto a los resultados obtenidos por la variable de género se identificó que existen diferencias entre los estudiantes de género femenino y los de género masculino en los factores orientación al ego, clima de aprendizaje por el profesor, clima de aprendizaje por los estudiantes y preocupaciones de los estudiantes por los errores. Así pues, el género se convierte en una variable moduladora de las orientaciones de meta y la percepción del clima motivacional, que a su vez debe ser tenida en cuenta por el docente para orientar la práctica deportiva. 
Finalmente, los resultados en cuanto a la comparación por género coinciden con los hallazgos obtenidos en otras investigaciones en donde los hombres tienen una mayor orientación al ego y preocupación por los errores, que las mujeres; éstas últimas se orientan más al clima de aprendizaje por el profesor y por el estudiante, lo cual puede estar relacionado con la percepción de un trato de igualdad por parte del docente como se señala en los estudios en Flórez, Salguero y Márquez (2008).

\section{REFERÊNCIAS}

ALMAGRO, B.; SAÉNZ-LÓPEZ, P.; GONZÁLEZ-CUTRE, D.; MORENO-MURCIA, J. Clima motivacional percibido, necesidades psicológicas y motivación intrínseca como predictores del comportamiento deportivo en adolescentes. Revista Internacional de Ciencias del Deporte, 25(7), p. 250-265, 2011.

AMES, C. Classrooms: goals, structures, and student motivation. Journal of Educational Psychology, (84), p. 261-271, 1992.

CASTILO, I.; BALAGUER, I.; Duda, J. Las orientaciones de meta y los motivos de práctica deportiva en los jóvenes deportistas valencianos escolarizados. Revista de Psicología del Deporte, (9), p. 37-50, 2000.

CECCHINI, J.; GÓNZLÁLEZ, C.; CARMONA, A.; CONTRERAS, O. Relaciones entre clima motivacional, la orientación de meta, la motivación intrínseca, la auto-confianza, la ansiedad y el estado de ánimo en jóvenes deportista. Psicothema, 16(1), p. 104-109, 2004.

CERVELLÓ, E.; JIMÉNEZ, R.; Del VILLAR, F.; RAMOS, L.; SANTOS-ROSA, F. J. Goal orientations, motivational climate, equality, and discipline of Spanish physical education students. Perceptual and Motor Skills, 99, p. 271-283, 2004.

\section{CERVELLÓ, E.; JIMÉNEZ, R. Validación de un instrumento para medir la percepción} del clima motivacional en las clases de Educación Física. En prensa, 2002.

DUDA, J. Ejercicio físico, motivación y salud, 2001. In: DEVIS, J. (coord.). La Educación Física, el Deporte y la Salud en el siglo XXI. Alcoy: Marfil, 2001.

DUDA, J.; WHITEHEAD, J. Measurement of goal perspectives in the physical domain, 1998. In: DUDA, J. L. (coord.). Advances in Sport and Exercise Psychology

Measurement. Fitness Information Technology, Inc., 1998.

FLÓREZ, J.; SALGUERO, A.; MÁRQUEZ, S. Relación de género, curso y tipo de colegio con el clima motivacional percibido en la Educación Física escolar en estudiantes colombianos. Revista de Educación. (37), p. 203-227, 2008.

GONZÁLEZ, E. Beneficios de la Educación Física y el deporte en los escolares. Innovación y Experiencias Educativas, 2007.

GUZMÁN, J.; GARCÍA-FERRIOL, A. Orientación de meta de los entrenadores y metodología de entrenamiento: implicaciones motivacionales. Revista Motricidad, (9), p. 65-82, 2002. 
HARWOOD, C.; HARDY, L. Persistence and effort in moving achievement goal research forward: A response to Treasure and colleagues. Journal of Sport and Exercise Psychology, (23), p. 330-345, 2001.

HERNÁNDEZ SAMPIERI, R.; FERNÁNDEZ-COLLAD, C.; BAPTISTA, L. P. Metodología de la investigación. México: McGraw Hill. Editores, 2014.

KOHLER, J.; REYES, M. Teoría de orientación a la meta: hallazgos y aplicaciones en la educación y la Educación Física. Revista Cultura, (24), p. 1-23, 2010.

LLANOS, C.; TABERNERO, B. Un estudio correlacional entre la orientación motivacional, el clima motivacional percibido y las creencias de éxito en las clases de Educación Física, 2003. In: MÁRQUEZ, S. (coord.). Psicología de la Actividad Física y el Deporte:

Perspectiva Latina. León: Universidad de León, 2003.

MADRIGAL, A. G.; URREGO, A. T. La Educación Física renovada desde la investigación educativa. Revista Lasallista de Investigación. 10(2), p.69-76, 2013.

McMILLAN, J.; SCHUMACHER, S. Investigación educativa. 5 ed., Pearson, 2005.

NICHOLLS, J. G. Achievement motivation: Conceptions of ability, subjective experience, task choice, and performance. Psychological Review, (91), p. 328-346, 1984.

NICHOLLS, J. G. The competitive ethos and democratic education. Cambridge, MASS: Harvard University Press, 1989.

NUNNALY, J. C. Psychometric theory. New York: McGraw-Hill Inc., 1978.

PAPAIOANNOU, A.; KOULI, O. The effect of task structure, perceived motivational climate, and goal orientations on students` task involvement and anxiety. Journal of Applied Sport Psychology, (11), p. 51-71, 1999.

PEIRÓ, C. La teoría de las perspectivas de meta y la Educación Física: Un estudio sobre los climas motivacionales. Revista de Psicología Social Aplicada, (9), p. 25-44, 1999.

PEIRÓ, C.; SANCHIS, J. Las propiedades psicométricas de la versión inicial del cuestionario de orientación a la tarea y al ego (TEOSQ) adaptado a la Educación Física en su traducción al castellano. Revista de Psicología del Deporte, 13(1), p. 25-39, 2004.

ROBERTS, G. C. Understanding the dynamics of motivation in physical activity: The influence of achievement goals on motivational processes, 2001. In: ROBERTS, G. C. (coord.). Advances in Motivation in Sport and Exercise. Champaign, Il: Human Kinetics, 2001.

ROBERTS, G. C.; TREASURE, D. C.; KAVUSSANU, M. Orthogonality of achievement goals and its relationship to beliefs about success and satisfaction in sport. The Sport Psychologist, (10), p. 398-408, 1996.

RODRÍGUEZ GARCÍA, P. L.; SÁNCHEZ LÓPEZ, C.; VALVERDE PUJANTE, J.; LÓPEZ MIÑARRO, P. A.; GARCÍA CANTÓ, E. Actividades físico-deportivas realizadas durante el tiempo de ocio por adolescentes escolarizados de la región de Murcia (España). Revista de Estudios y Experiencias en Educación, (10), p. 13-32, 2011. 
RUÍZ, J.; PIÉRON, M.; ZAMARRIPA, J. Versión española del Task and Ego Orientation in Sport Questionnaire (TEOSQ) adaptado a Educación Física. Estudios de Psicología, 32(2), p. 179-193, 2011.

SÁNCHEZ-OLIVIA, D.; LEO, F.; SÁNCHEZ-MIGUEL, P.; AMADO, D.; GARCÍACALVO, T. Relación del clima motivacional creado por el entrenador con la motivación auto-determinada y la implicación hacia la práctica deportiva. Revista Internacional de Ciencias del Deporte, 2010.

WALLING, M.; DUDA, J. Goals and their associations with belief about success in and perceptions of the purposes of physical education. Journal of Teaching in Physical Education, (14), p. 140-156, 1995. 